\title{
Novel Femto Laser Patterning of High Translucent Zirconia as an Alternative to Conventional Particle Abrasion
}

\author{
Emmanouil-George C. Tzanakakis ${ }^{1}(\mathbb{D})$, Anastasia Beketova ${ }^{2} \mathbb{D}$, Lambrini Papadopoulou ${ }^{3}(\mathbb{D}$, \\ Eleana Kontonasaki ${ }^{2,4, *(D)}$ and Ioannis G. Tzoutzas ${ }^{1}$ (D) \\ 1 School of Dentistry, National and Kapodistrian University of Athens, 2 Thivon Str, Goudi, \\ 11527 Athens, Greece; tzanakak@dent.uoa.gr (E.-G.C.T.); tzoudent@dent.uoa.gr (I.G.T.) \\ 2 Department of Prosthodontics, School of Dentistry, Faculty of Health Sciences, \\ Aristotle University of Thessaloniki, 54124 Thessaloniki, Greece; anastasiabeketova@yahoo.com \\ 3 School of Geology, Faculty of Sciences, Aristotle University of Thessaloniki, 54124 Thessaloniki, Greece; \\ lambrini@geo.auth.gr \\ 4 Center for Interdisciplinary Research and Innovation (CIRI-AUTH), Balkan Center, \\ 57001 Thessaloniki, Greece \\ * Correspondence: kont@dent.auth.gr
}

Citation: Tzanakakis, E.-G.C.;

Beketova, A.; Papadopoulou, L.; Kontonasaki, E.; Tzoutzas, I.G. Novel Femto Laser Patterning of High Translucent Zirconia as an Alternative to Conventional Particle Abrasion. Dent. J. 2021, 9, 20. https://doi.org/10.3390/dj9020020

Academic Editor: Samir Nammour Received: 30 December 2020 Accepted: 4 February 2021

Published: 8 February 2021

Publisher's Note: MDPI stays neutral with regard to jurisdictional claims in published maps and institutional affiliations.

Copyright: (c) 2021 by the authors. Licensee MDPI, Basel, Switzerland. This article is an open access article distributed under the terms and conditions of the Creative Commons Attribution (CC BY) license (https:// creativecommons.org/licenses/by/ $4.0 /)$.
Abstract: Background: currently applied surface treatments for zirconia bonding may create undesired microcracks and surface flaws. The aim of the present study was to evaluate the efficacy of alternative surface treatments on the shear bond strength of high translucency zirconia to 10Methacryloyloxydecyl dihydrogen phosphate (MDP)-containing resin-based cement. Methods: fifty disk-shaped specimens $(10 \mathrm{~mm} \times 5 \mathrm{~mm})$ were fabricated from a commercial yttria-stabilized zirconia with 5 mole\% yttrium oxide tetragonal zirconia polycrystal (5Y-TZP), and underwent air-abrasion with alumina particles (50 $\mu \mathrm{m}$-AL50 and $90 \mu \mathrm{m}$-AL90), glass beads (GB 10-60 $\mu \mathrm{m}$ ), and ablation with femtosecond laser (FEMTO). Shear bond strength was evaluated with a universal testing machine under a crosshead speed of $0.5 \mathrm{~mm} / \mathrm{min}$ until fracture. Fracture type was evaluated with an optical stereomicroscope. Differences among groups were evaluated by one-way ANOVA and Bonferroni pairwise comparison tests $(p<0.05)$. Results: the highest shear bond strength values were presented by the laser treated group $(23.97 \pm 3.7 \mathrm{MPa})$. No statistically significant differences were found among the $\mathrm{Cl}, \mathrm{Al} 50, \mathrm{~A} 190$ and FEMTO groups. The lowest mean value was presented by the glass-beads treated group $(11.93 \pm 2.88 \mathrm{MPa})$ which was significantly lower compared to all other groups $(p<0.001)$. Conclusions: under the limitations of this in vitro study, femtosecond laser treatment of High-translucent monolithic zirconia (HTZ) ceramics is a promising alternative method for the mechanical retention of resin cements.

Keywords: zirconia ceramic; resin cement; shear bond strength; laser; surface treatment

\section{Introduction}

In recent years, dental manufacturers have introduced a new generation of zirconia ceramics that are suitable for tooth reconstruction in the esthetic zone. High-translucent monolithic zirconia (HTZ) has gained much interest from patients with esthetic demands because of its superior optical properties. In clinical practice, high-translucent zirconia has been used to produce monolithic anterior crowns and ultrathin restorations. Its higher translucency is achieved by a slight increase in the $\mathrm{Y}_{2} \mathrm{O}_{3}$ content (higher than 3 mol-\%), which also increases the percentage of the transparent cubic-phase [1,2]. On the other hand, HTZ materials possess significantly lower flexural strength (550-800 MPa instead of 900-1400 MPa), and even poorer adhesive behavior with resin based cements as compared to conventional zirconia [3-5]. Decementation of crowns, especially in the anterior area, can compromise the whole clinical outcome and cause patient dissatisfaction, whereas in case of ultrathin HTZ restorations, cementation failure can easily result in chipping or fracture, as resin bonding only provides them with limited strength. 
There are limited scientific data about the bonding strength of translucent zirconia subjected to different surface treatments. As other types of zirconia, HTZ possesses a dense polycrystalline structure with no vitreous phase, which makes its surface processing quite complicated. Methods such as airborne abrasion with alumina particles [6], tribochemical silica coating [7], deposition of low fusing porcelain [8], plasma coating with hexamethyldisiloxane [9], laser surface ablation [10,11], selective infiltration etching [12], and zirconia primers/adhesives [13] and many others have been proposed for conventional zirconia.

Currently, the most reliable bonding protocols for zirconia is the combination of air-abrasion with alumina $\left(\mathrm{Al}_{2} \mathrm{O}_{3}\right)$ or silica-coated alumina $\left(\mathrm{Al}_{2} \mathrm{O}_{3} / \mathrm{SiO}_{2}\right)$ particles and further surface conditioning with an MDP-containing primer [14,15]. Abrasive alumina particles of different sizes are used to remove impurities and increase its surface roughness, thereby providing mechanical interlocking with cement. In turn, MDP-containing primer can chemically bond to the zirconia surface through zirconium phosphate formation. Recently, Salem et al. [16] reported promising results for translucent zirconia adhesive strength when using alumina air-particle abrasion combined with an MDP-containing adhesive. Another technique, air abrasion with silica-coated alumina particles, known as tribochemical silica coating, leaves the zirconia surface with a thin silica layer that is able to react with silane. In this respect, Ruales-Carrera et al. [5] found that tribochemical treatment with $\mathrm{Al}_{2} \mathrm{O}_{3} / \mathrm{SiO}_{2}$ particles was effective at increasing the bond strength of both conventional and highly translucent zirconia. On the other hand, recent in vitro studies reported that airborne-particle abrasion with coarse particles may create microcracks, initiating fractures on the zirconia surface $[17,18]$. For this reason, there is a strong need for investigation of alternative surface conditioning methods for zirconia, particularly, for the more brittle HTZ materials.

Recently, it has been reported that air blasting of the zirconia surface with glass beads can improve the bond strength between zirconia and resin cement [19]. The fusion of glass particles on a zirconia surface has a two-fold effect; it creates a chemical bond between zirconia and cement through the silane bonding and modifies surface topography increasing bond strength $[9,20]$. However, airborne abrasion with glass beads has not been applied to HTZ materials so far.

Another promising method for modifying the zirconia surface, is application of a laser. Laser surface treatment is rapid, non-contact and precise, as it allows one to obtain high-resolution features, down to the nanoscale. Several short pulse lasers such as Nd:YAG, Er:YAG, and Er,Cr:YSGG continuous wave carbon dioxide $\left(\mathrm{CO}_{2}\right)$, have been suggested for zirconia surface treatment [21-23]. However, Er:YAG and $\mathrm{CO}_{2}$ lasers can cause surface microcracking $[22,24]$ which might reduce the flexural strength. On the other hand, femtosecond lasers can produce ultrashort pulses of high intensity and ablate material only superficially, without causing thermal damage. To achieve a desired surface pattern, different types of surface craters can be created through appropriate software. Femtosecond laser application creates rough zirconia surfaces without triggering phase transformations $[25,26]$. Moreover, in our previous study it was demonstrated that femtosecond laser patterning of HTZ surface resulted in a rough surface pattern in laser affected areas and the laser-induced grooves presented a repetitive morphology with parallel lines [27].

Based on the aforementioned, the aim of the present study was to evaluate the efficacy of novel surface treatments-air-particle abrasion with glass beads and femtosecond laser ablation-on the shear bond strength of HTZ to resin-based cement. The research hypothesis was that different surface treatments provide the same shear bonding strength and fracture mode between zirconia and resin-based cement.

\section{Materials and Methods}

For the experiments, white colored blocks of commercial yttria-stabilized zirconia 5Y-TZP (92\% $\mathrm{ZrO}_{2}, 5.2 \% \mathrm{Y}_{2} \mathrm{O}_{3}, \mathrm{HfO}_{2}<4 \%$, and $\mathrm{Al}_{2} \mathrm{O}_{3}<0.5 \%, \mathrm{SiO}_{2}<1 \%$ ) (Bruxzir HT 2.0, Glidewell, Newportbeach, CA, USA) (LOT HT-BZ0014192) were used. For air abrasion, $\mathrm{Al}_{2} \mathrm{O}_{3}$ particles of size $50 \mu \mathrm{m}$ (Luoyang, Yannuo, China), $\mathrm{Al}_{2} \mathrm{O}_{3}$ particles with size $90 \mu \mathrm{m}$ 
(Danville San Ramon, CA, USA) and glass beads (Luoyang, Yannuo, China) with particle diameter of 10-60 $\mu \mathrm{m}$ were used. Commercially available methylacrylate-based dual-cured resin cement Panavia V5 together with Clearfil ceramic primer plus (Kuraray, Okayama, Japan) were chosen as experimental materials because they incorporate MDP functional monomers.

Fifty disk-shaped specimens with diameter $10 \mathrm{~mm}$ and height $5 \mathrm{~mm}$ were fabricated using Computer Aided Design/Computer Aided Manufacturing (CAD/CAM). Then, the specimens were impregnated in epoxy resin (Epofix, Struers, Denmark) using Plexiglas molds of $25 \mathrm{~mm}$ height, $12 \mathrm{~mm}$ internal diameter and $16 \mathrm{~mm}$ external diameter and were kept for $12 \mathrm{~h}$ under atmospheric pressure until the complete polymerization of epoxy resins. After removal of the specimens from the molds they were ground in a rotary motion metallurgical grinding apparatus at speed of $200 \mathrm{rpm}$ and water irrigation. Grinding and polishing of specimens was performed under constant pressure using metallic holder with 220 and 1200 grit silicon carbide grinding discs for $10 \mathrm{~min}$. In order to check for surface defects during construction and processing, each specimen was examined using a stereo microscope (M80, Leica, Weltzar, Germany) connected to a PC with the appropriate software at magnifications of $7.5 \times$ to $60 \times$. Specimens with surface defects were removed and replaced with new ones. Finally, all specimens were ultrasonically cleaned in a pure ethyl alcohol for $15 \mathrm{~min}$, dried with a mild air stream and divided into 5 equal groups $(\mathrm{N}=10)$ based on surface treatment, as following:

(a). CL group: Control group with no further processing after polishing;

(b). AL50 group: air-abraded by $50 \mu \mathrm{m}$ alumina particles;

(c). AL90 group: air-abraded by $90 \mu \mathrm{m}$ alumina particles;

(d). GB group: air-abraded by glass beads of various diameters of 10-60 $\mu \mathrm{m}$;

(e). FEMTO group: in which parallel grooves of $50 \mu \mathrm{m}$ in the central area of $5 \times 5 \mathrm{~mm}$ were processed by femtosecond laser ablation.

The air abrasion process for the AL50, AL90 and GB groups was performed using an intraoral sandblaster device (Microetcher IIA, Danville, CA, USA), equipped with a 0.048 diameter nozzle, at the air pressure $36 \mathrm{psi}(2.5 \mathrm{bar})$ for $10 \mathrm{~s}$ and distance of $10 \mathrm{~mm}$ and incidence angle of $45^{\circ}$ to the free zirconia surface. A special holder was fabricated to maintain exact distance and incidence angle in all air abraded specimens.

For the experiments, a Yb: KGW laser was used, emitting linearly polarized light with central wavelength of $1026 \mathrm{~nm}$, pulse duration $170 \mathrm{fs}$ and repetition rate of $1 \mathrm{KHz}$. The laser beam was focused on the sample through $100 \mathrm{~mm}$ achromatic convex lens while the estimated Gaussian spot size, had $35 \mu \mathrm{m}$ diameter, as measured with a CMOS camera. The zirconia samples were mounted in a 3-axis support and movement device and placed vertically to the incident beam. Laser radiation was emitted under normal environmental conditions with normal incidence direction. The designed surface pattern included an area of $5 \mathrm{~mm} \times 5 \mathrm{~mm}$ and consisted of continuous horizontal line scans with $50 \mu \mathrm{m}$ line spacing. For the surface processing the zirconia samples the laser parameters were: laser fluence, $\mathrm{F}=9.6 \mathrm{~J} / \mathrm{cm}^{2}$ and sample scanning speed, $\mathrm{v}=1 \mathrm{~mm} / \mathrm{s}$.

At first, each specimen was coated by one component adhesive primer (Clearfil Ceramic Primer plus) using an applicator brush, as described by the manufacturer. For repeatability and precision of the bonding procedures, five special two-piece teflon guides with a center hole $(3 \times 3 \mathrm{~mm})$ were constructed for zirconia specimens. Then, the resin-based cement (Panavia V5) was injected using self-mixing syringe in the center of each teflon guide until the empty space was filled. After $1 \mathrm{~min}$ the cement was photo-activated using light emitting diode-LED (T-LED, Anthos, Imola, Italy) with output power $800 \mathrm{~mW} / \mathrm{cm}^{2}$ for $20 \mathrm{~s}$. After polymerization and complete removal of the teflon guides, the specimens were kept in deionized water at $37^{\circ} \mathrm{C}$ in a temperature controlling apparatus for one week.

For shear bond strength test a universal testing machine (Monsanto Tensometer 10, Swindon, UK) was used operating in shear mode with a crosshead speed of $0.5 \mathrm{~mm} / \mathrm{min}$ until fracture of the zirconia/cement interface. Maximum values of shear strength (peak points), expressed as $\mathrm{N}$, were recorded for each specimen. In order to calculate the maximum shear 
stress, these values were conversed to $\mathrm{MPa}$, using the equation $\mathrm{P}=\mathrm{F} / \mathrm{S}$, where $\mathrm{F}$ is the force in Newton $(N)$ and $S=\pi R^{2}$ the bonded surface.

For the evaluation of the type of fracture, the detached surfaces were photographed using an optical stereomicroscope at magnifications of 10-60 X. Quantification of the remaining ceramic mass or resin-based cement on the surface of zirconia was done with the help of a PC software program (Adobe Systems, San Jose, CA, USA). Fracture was defined as adhesive in cases where more than $75 \%$ of the zirconia surface was visible. The fracture was defined as cohesive when more than the $75 \%$ of the core surface was covered with resin. All other cases were defined as mixed fractures. For further evaluation of the debonded surfaces and elemental analysis, Scanning Electron Microscopy (JEOL J.S.M. 840A, Tokyo, Japan) and Energy Dispersive Spectroscopy (Oxford INKA-300) were applied. A vacuum evaporator (JEOL-4X) was used to coat the surfaces with a carbon coat of $200 \AA$ for SEM-EDS evaluation. Shear bond strength values were analyzed for normality and homogeneity of variance with Kolmogorov-Smirnof and Levene tests, respectively, while differences among groups were evaluated by one-way ANOVA and Bonferroni multiple comparison test. The results of failure type were also were also evaluated with one-way ANOVA. Statistical analysis was performed with the IBM Statistics SPSS 20.0 software with significance level set at $p<0.05$.

\section{Results}

The results of the maximum shear strength and the standard deviations in $\mathrm{MPa}$ of the five experimental groups are presented in Figure 1. The ranking of groups in decreasing shear bond strength were: FEMTO > AL90, AL50, CL > GB.

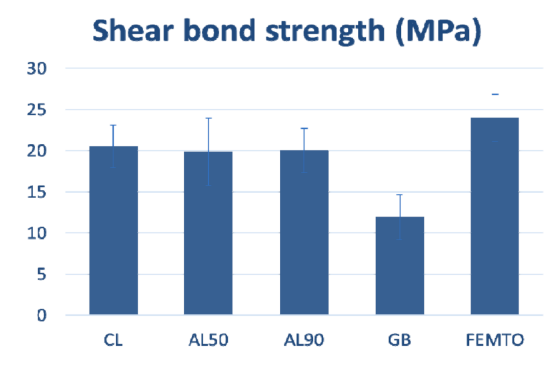

Figure 1. Mean values and standard deviation (bars) of shear bond strength values.

According to the ANOVA analysis, no statistically significant differences were found among the Cl, AL50, AL90 and FEMTO groups (F: 16.638, df: 4, $p<0.001$ ). The lowest mean value was recorded for the GB group $(11.93 \pm 2.88 \mathrm{MPa})$ which was statistically significantly different compared with all other groups $(p<0.001)$ (Table 1$)$.

Table 1. Bonferroni Multiple Comparison tests of shear bond strength values among the groups.

\begin{tabular}{|c|c|c|c|c|c|c|}
\hline \multicolumn{7}{|c|}{ Bonferroni Multiple Comparisons (Dependent Variable: Shear Bond Strength) } \\
\hline \multirow{2}{*}{ (I) TREATMENT } & \multirow{2}{*}{ (J) TREATMENT } & \multirow{2}{*}{ Mean Difference (I-J) } & \multirow{2}{*}{ Std. Error } & \multirow{2}{*}{ Sig. } & \multicolumn{2}{|c|}{ 95\% Confidence Interval } \\
\hline & & & & & Lower Bound & Upper Bound \\
\hline \multirow{4}{*}{ CL } & FEMTO & -3.444 & 1.536 & 0.299 & -7.979 & 1.091 \\
\hline & AL50 & 0.692 & 1.536 & 1.000 & -3.842 & 5.227 \\
\hline & AL90 & 0.488 & 1.536 & 1.000 & -4.047 & 5.023 \\
\hline & GB & $8.601 *$ & 1.536 & $<0.0001$ & 4.066 & 13.136 \\
\hline \multirow{3}{*}{ FEMTO } & AL50 & 4.136 & 1.536 & 0.099 & -0.399 & 8.671 \\
\hline & AL90 & 3.932 & 1.536 & 0.139 & -0.602 & 8.467 \\
\hline & GB & 12.045 * & 1.536 & $<0.0001$ & 7.510 & 16.579 \\
\hline \multirow{2}{*}{ AL50 } & AL90 & -0.204 & 1.536 & 1.000 & -4.739 & 4.331 \\
\hline & GB & $7.909 *$ & 1.536 & $<0.0001$ & 3.374 & 12.444 \\
\hline AL90 & GB & 8.113 * & 1.536 & $<0.0001$ & 3.578 & 12.648 \\
\hline
\end{tabular}

* The mean difference is significant at the 0.05 level. 
The highest mean shear bond strength values were presented by the FEMTO group $(23.97 \pm 3.7 \mathrm{MPa})$ which was statistically significantly different only compared to the GB group $(p<0.001)$. According to adhesive failure modes (ADFM) analysis FEMTO, $\mathrm{Cl}$, AL50 and AL90 groups presented significantly lower ADFM values than the GB group (Table 2).

Table 2. Percentages of adhesive-type of failure (ADFM).

\begin{tabular}{ccc}
\hline Group & ADFM (\%) \\
\hline CL & $20 \% \mathrm{a}^{*}$ \\
AL50 & $30 \% \mathrm{a}$ \\
AL90 & $30 \%$ a \\
GB & $80 \% \mathrm{~b}$ \\
FEMTO & $20 \% \mathrm{c}$ \\
\hline
\end{tabular}

* Similar Latin characters indicate non-statistically significant differences between groups.

SEM-EDS analysis showed the surface pattern composed of parallel grooves filled with resin-based cement of the FEMTO group specimens (Figure 2).
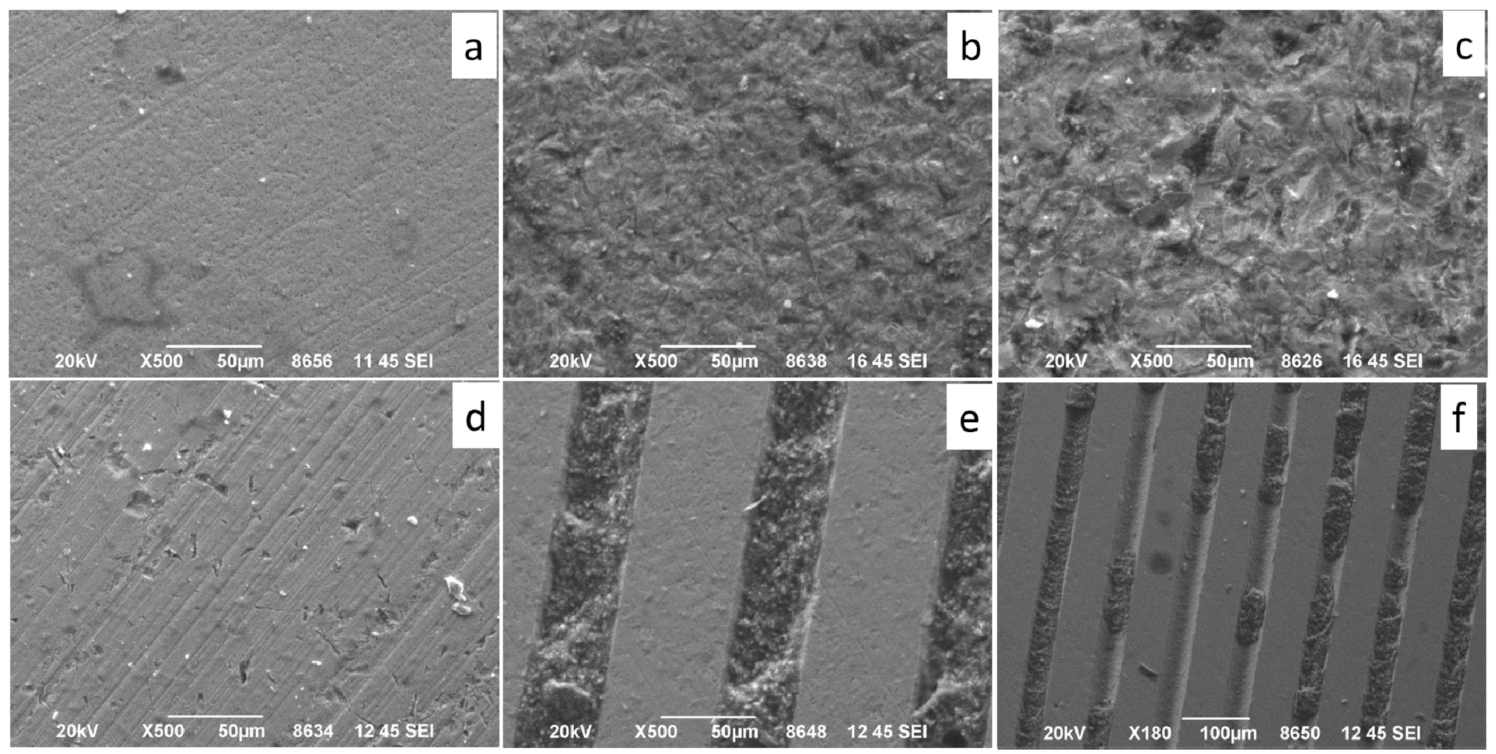

Figure 2. SEM microphotographs of fractured surfaces. (a) control (CL group), (b) air-borne microparticles of $50 \mu \mathrm{m}$ alumina (AL50 group). (c) air-borne microparticles of $90 \mu \mathrm{m}$ alumina (AL90 group), (d) air-borne microparticles of glass beads (GB group) and (e) laser pattern of parallel grooves of $50 \mu \mathrm{m}$ (FEMTO group), (f) lower magnification microphotograph of FEMTO group showing cement remnants in all microgrooves.

The SEM microphotographs of AL90 and AL50 samples show irregular rough surface with residues of cement on it (Figure 3). The surface of AL90 appeared to be rougher as compared to the specimens of AL50 group, GB and CL. EDS analysis showed the presence of $\mathrm{Al}_{2} \mathrm{O}_{3}$-rich phase entrapped within the surface irregularities in AL50 and AL90 specimens. Finally, the surface roughness GB treated specimens remained unchanged and similar to the control group, while the presence of small superficial Si-containing particles was detected by EDS (Figure 3). 


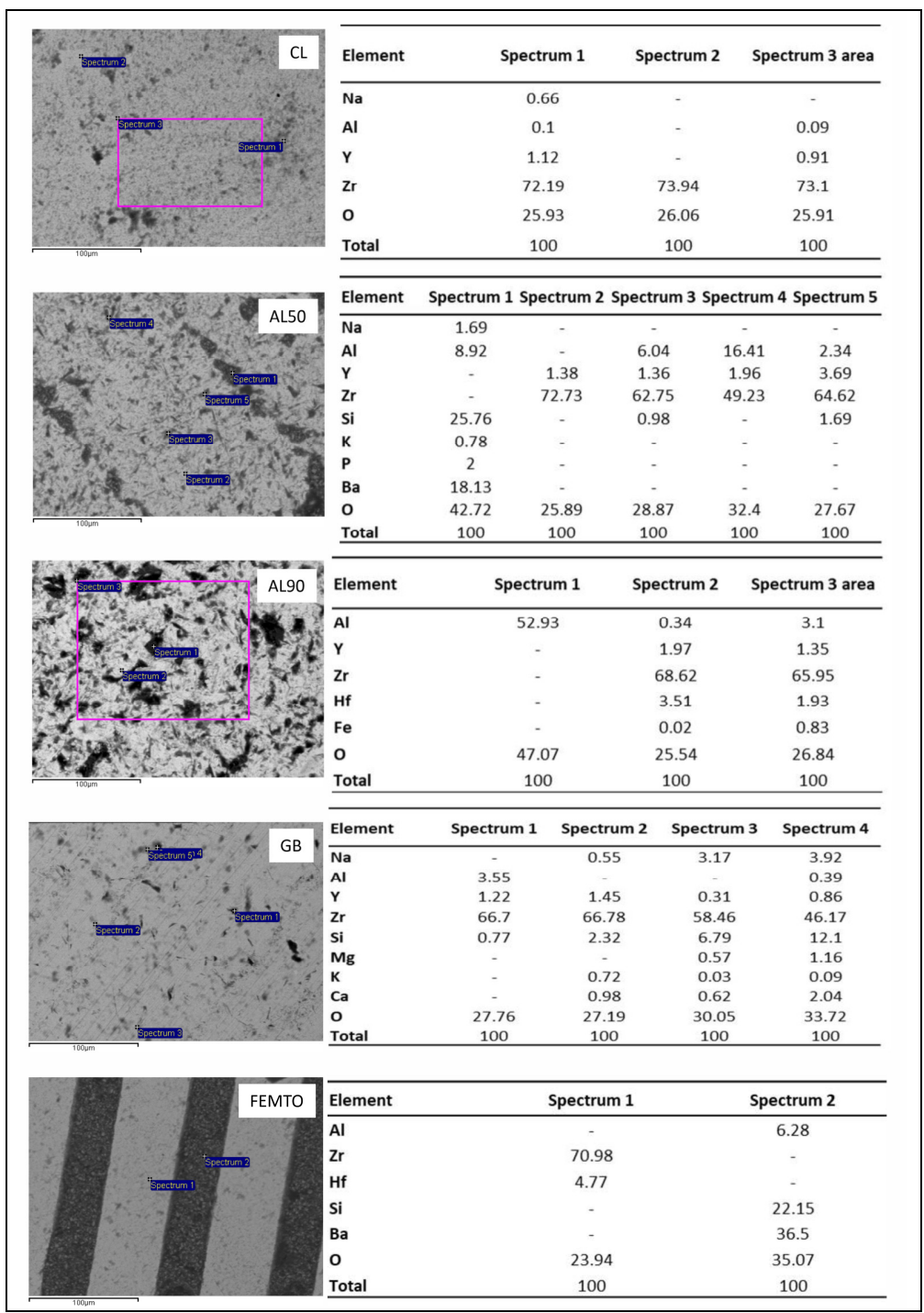

Figure 3. Left: SEM Backscattered microphotographs. Right: EDS compositional analysis from the respec-tive microphotographs.

\section{Discussion}

In the presented study five different surface treatments were applied to novel hightranslucent zirconia and shear bonding strength with resin-based cement was evaluated. The results showed that the GB group presented statistically significant differences compared with the FEMTO, CL, AL50, AL90 groups in shear bond strength and fracture type, and that there were no statistically significant differences among specimens of FEMTO, CL, AL50 and AL90 groups, thereby partially rejecting the research hypothesis.

The highest average values of shear bond strength $(23.97 \pm 3.7 \mathrm{MPa})$ were observed in specimens of the FEMTO group, which presented a moderated surface roughness of $307.3 \pm 32.7 \mathrm{~nm}$ [27], which was similar to AL50 (AL50 = 340.3 $\pm 49.2 \mathrm{~nm}$ ) but significantly higher compared to $\mathrm{CL}(\mathrm{CL}=73.9 \pm 10.7 \mathrm{~nm})$ [27]. Similar results were obtained by Vicente Prieto et al. [28], who reported superior values of Shear Bond Strength (SBS) in specimens that underwent femto-laser surface patterning as compared with airborneparticle abrasion (APA) and tribochemical coating of conventional zirconia. At the same time, the FEMTO group also presented mostly mixed type fracture, and similar percentage of adhesive failure modes (ADFM) compared with CL, AL50 and AL90 groups. In fact, the SEM analysis presented penetration of the cement inside the grooves and adhesive decementation at all other areas. Moreover, the presence of the element barium $(\mathrm{Ba})$ in 
high percentage (36.5\%) proves the high percentage of resin cement in the laser groove area. The results of the fracture type suggest that the laser treated surface resulted in mechanical interlocking that was responsible for the high acquired SBS values. However, as seen in lower magnification (Figure 3e), some areas in the grooves were not covered by resin cement. One possible explanation is that the rheological properties of the resin cement hampered the complete penetration of the material in the grooves. In addition, inhomogeneous diffusion of the primer inside the retentive laser lines may have contributed to this result. This hypothesis is also supported by the unexpectedly high shear strength values of the control group which are mainly based on chemical activation rather than micromechanical retention. The depth, width and roughness of the retentive grooves need further evaluation to define the optimum conditions. Additionally, shallower or wider grooves could perhaps allow better flow of the primer. Another explanation is that cohesive strength of the resin cement surpassed the shear bond adhesive potential and resin cements remnants could have been detached during shear stressing.

Processing of HTZ in femtosecond regime enabled precise micropatterning and creation of parallel retention microgrooves, to obtain extra space for cement. SEM images revealed increased microroughness within the grooves due to ablation while the untreated surface remained smooth and free of microcracks, pores and signs of melting. These findings suggest that the ultrashort pulses with high intensity applied in the present study caused ablation only within laser generated plasma, without causing thermal damage to the adjacent regions due to heat conduction; a phenomenon usually observed with conventional lasers (nanosecond or longer pulses) [25].

According to recent studies, both irradiation patterns [29] and different angulations $[26,30]$ of the laser affect the bond strength between the ceramic surface and resinbased material. Vincente et al. [31] produced two uniform surface patterns with parallel grooves with depth of $20 \mu \mathrm{m}$ using scanning steps of 20 and $40 \mu \mathrm{m}$ and found no significant differences in shear bond strength between groups. In accordance with our results, the authors observed mostly mixed types of failures. Akpinar et al. [26] used different beam angles $\left(45^{\circ}, 60^{\circ}, 75^{\circ}\right.$ and $\left.90^{\circ}\right)$ on zirconia surfaces and reported smaller bond strength values with the $90^{\circ}$ beam angle. Yucel et al. [30] also reported better results with the $30^{\circ}$ laser beam angle, which were attributed to more retentive areas with this beam inclination that resulted in an increase in the total contact area. So far, further improvements in the bond strength of laser processed HTZ ceramics could be achieved by optimization of laser surface patterning.

Air-borne particle abrasion was applied to HTZ using alumina particles of different sizes $(50 \mu \mathrm{m}$ and $90 \mu \mathrm{m})$ and GB. The AL50 and AL90 groups had similar bond strength values and similar types of fracture, covering a high percentage of the zirconia surface with resin material, suggesting that surface roughness profile did not significantly affect the shear strength. The obtained values of bond strength for alumina air-borne treated groups (AL50 and AL90) were comparable to other studies with high-translucent zirconia $[5,16]$. It was expected that alumina abrasion with particles of larger size would result in a rougher surface, assisting micromechanical retention [32]. Indeed, the SEM micrographs and roughness measurements reported in our previous study showed that AL90 specimens had rougher surfaces (AL50 $=340.3 \pm 49.2 \mathrm{~nm}$ ) as compared to AL50 $(\mathrm{AL90}=1155.1 \pm 97.76 \mathrm{~nm})$ [27]. EDS analysis showed the increased $\mathrm{Al}$ and $\mathrm{O}$ content in both groups, probably due to the inclusion of $\mathrm{Al}_{2} \mathrm{O}_{3}$ particles onto the ceramic surfaces during alumina air-abrasion. However, statistically significant differences in SBS between AL50 and AL90 groups were not observed. In this way, the surface roughness profile from the different alumina particle sizes did not influence the bonding strength in the HTZ material, similarly to conventional zirconia, as previously reported [33].

The GB group presented very low shear strengths and very small percentages of the resin material remained on the surface, resulting in a high percentage of adhesive fracture mode. Based on our previous study and SEM analysis, it was observed that GB created a smooth surface with slightly higher toughness than the control $(\mathrm{GB}=99.4 \pm 16.6 \mathrm{~nm}$, 
$\mathrm{CL}=73.9 \pm 10.7 \mathrm{~nm}$ ) [27], likely because glass particles are much softer than alumina or zirconia. Therefore, another mechanism, irrespective of roughness, was responsible for these values. Possible contamination of the zirconia surface by the glass beads or incompatibility between them and the adhesive primer could explain the vertical drop in shear strength in this group, compared to CL that presented even lower surface roughness. Probably, very small glass beads-detected by EDS—could remain loose on the zirconia surface and interfere with the action of the primer's phosphate monomers. As it was recently reported by Nagaoka group, zirconia surface contamination by residual silica particles may inhibit adequate bonding of the cement to zirconia [34]. On the contrary, Martins et al. [19] observed enhanced zirconia/resin cement bonding strength after air blasting with glass beads. The favorable results of this study might be explained by initial alumina air blasting and application of silane coupling agent for all specimens that was not performed in the present study.

Bonding is known to be affected by many factors, such as micro-mechanical retention, chemical adhesion, surface features, and the type of adhesives [35]. Recently, Yagawa et al. [36] tested the effect of different priming methods on bond strength between luting cements and a translucent zirconia material, without any micromechanical surface pre-treatment. It was found that the application of MDP priming agents created a durable bond strength between resin cements and translucent zirconia [36]. In the present study, we also applied an MDP-containing primer to achieve enhanced bonding. Our findings suggest that non-mechanically treated control specimens had a similar bonding ability to AL50 and AL90 groups and low percentage of adhesive failures. Low roughness and lack of surface contamination might have favored better surface wetting and thus could have improved the development of chemical bonds between zirconia primer and resin-based cement. In these cases, the shear bond strength seems to depend more on the inherent cement strength rather than on the surface roughness or that on unobstructed chemical bonding, irrespective of surface roughness, is necessary for effective bonding.

Apparently, surface treatments such as air abrasion often leave traces of contaminants on the material's surface. Even though their amount is small, they can alter surface properties and compromise mechanical behavior as is the case with glass bead contamination. In this respect, ultrafast laser patterning is effective in creating an uncontaminated surface, as it can produce surfaces without any impurities. Besides, FS laser processing is a gentle and precise procedure, as it allows for the creation of different surface patterns without causing damage to the bulk material or significantly increasing the monoclinic phase [27], which makes it an appropriate method for HTZ ceramics surface conditioning. The feasibility to produce and alter zirconia surface patterning with the use of an FS laser is a wide-open field for future research, as many interesting retentive features can be created, with the aim of optimizing the bond strength.

Despite predictable and well-documented results, several limitations of this study should be mentioned. For example, the small number of experimental materials studied. Further research should include different HTZ materials with other commercial resin-based cements and conditions that mimic, with high precision, the oral environment such as cycling loading.

\section{Conclusions}

Under the limitations of this in vitro study, it can be concluded that airborne particle abrasion with alumina particles of different sizes $(50 \mu \mathrm{m}$ and $90 \mu \mathrm{m})$ and an FS laser, can yield similar bond strengths of HTZ ceramics to resin cements. However, none of the investigated treatments were superior to the others or compared to control polished zirconia surfaces. FS laser surface treatment of HTZ ceramics is a promising alternative method to enhance the mechanical retention of a resin cement as it is related to effective bond strengthening, without causing any surface contamination of zirconia specimens. On the contrary, abrasion with glass beads is not recommended for HTZ ceramics, as it presented significantly lower bond strengths compared to all other treatments. 
Author Contributions: Investigation and validation, E.-G.C.T.; writing-draft preparation, A.B.; visualization, A.B.; methodology, L.P., E.-G.C.T. and A.B., data curation, E.K.; writing-review and editing, E.K.; supervision, I.G.T.; project administration I.G.T.; funding acquisition E.-G.C.T. and I.G.T. All authors have read and agreed to the published version of the manuscript.

Funding: This research received no external funding.

Conflicts of Interest: The authors declare no conflict of interest.

\section{References}

1. Zhang, Y. Making yttria-stabilized tetragonal zirconia translucent. Dent. Mater. 2014, 30, 1195-1203. [CrossRef] [PubMed]

2. Zhang, F.; Inokoshi, M.; Batuk, M.; Hadermann, J.; Naert, I.; Van Meerbeek, B.; Vleugels, J. Strength, toughness and aging stability of highly-translucent Y-TZP ceramics for dental restorations. Dent. Mater. 2016, 32, e327-e337. [CrossRef] [PubMed]

3. Blatz, M.B.; Vonderheide, M.; Conejo, J. The Effect of Resin Bonding on Long-Term Success of High-Strength Ceramics. J. Dent. Res. 2018, 97, 132-139. [CrossRef] [PubMed]

4. Church, T.D.; Jessup, J.P.; Guillory, V.L.; Vandewalle, K.S. Translucency and strength of high-translucency monolithic zirconium oxide materials. Gen. Dent. 2017, 65, 48-52.

5. Ruales-Carrera, E.; Cesar, P.F.; Henriques, B.; Fredel, M.C.; Özcan, M.; Volpato, C.A.M. Adhesion behavior of conventional and high-translucent zirconia: Effect of surface conditioning methods and aging using an experimental methodology. J. Esthet. Restor. Dent. 2019, 31, 388-397. [CrossRef]

6. Blatz, M.B.; Phark, J.H.; Ozer, F.; Mante, F.K.; Saleh, N.; Bergler, M.; Sadan, A. In vitro comparative bond strength of contemporary self-adhesive resin cements to zirconium oxide ceramic with and without air-particle abrasion. Clin. Oral Investig. 2010, 14, 187-192. [CrossRef]

7. Elsaka, S.E. Influence of Surface Treatments on the Bond Strength of Resin Cements to Monolithic Zirconia. J. Adhes. Dent. 2016, 18, 387-395.

8. Cura, C.; Isik, G.; Saracoglu, A.; Özcan, M. Comparison of alternative adhesive cementation concepts for zirconia ceramic: Glaze layer vs zirconia primer. J. Adhes. Dent. 2012, 14, 75-82. [PubMed]

9. Derand, T.; Molin, M.; Kvam, K. Bond strength of composite luting cement to zirconia ceramic surfaces. Dent. Mater. 2005, 21, 1158-1162. [CrossRef]

10. Akin, H.; Tugut, F.; Akin, G.E.; Guney, U.; Mutaf, B. Effect of Er:YAG laser application on the shear bond strength and microleakage between resin cements and Y-TZP ceramics. Lasers Med. Sci. 2012, 27, 333-338. [CrossRef] [PubMed]

11. Kara, O.; Kara, H.B.; Tobi, E.S.; Ozturk, A.N.; Kilic, H.S. Effect of Various Lasers on the Bond Strength of Two Zirconia Ceramics. Photomed. Laser Surg. 2015, 33, 69-76. [CrossRef] [PubMed]

12. Aboushelib MN Evaluation of zirconia/resin bond strength and interface quality using a new technique. J Adhes. Dent. 2011, 13, 255-260.

13. Yoshida, K.; Tsuo, Y.; Atsuta, M. Bonding of dual-cured resin cement to zirconia ceramic using phosphate acid ester monomer and zirconate coupler. J. Biomed. Mater. Res. Part B Appl. Biomater. 2006, 77, 28-33. [CrossRef]

14. Tzanakakis, E.-G.C.; Tzoutzas, I.G.; Koidis, P.T. Is there a potential for durable adhesion to zirconia restorations? A systematic review. J. Prosthet. Dent. 2015, 115, 9-19. [CrossRef]

15. Ozcan, M.; Bernasconi, M. Adhesion to Zirconia Used for Dental Restorations: A Systematic Review and Meta-Analysis. J. Adhes. Dent. 2015, 17, 7-26.

16. Salem, R.; el Naggar, G.; Aboushelib, M.; Selim, D. Microtensile bond strength of resin-bonded hightranslucency zirconia using different surface treatments. J. Adhes. Dent. 2016, 18, 191-196. [PubMed]

17. Hallmann, L.; Ulmer, P.; Reusser, E.; Hämmerle, C.H.F. Effect of blasting pressure, abrasive particle size and grade on phase transformation and morphological change of dental zirconia surface. Surf. Coat. Technol. 2012, 206, 4293-4302. [CrossRef]

18. Zhang, Y.; Lawn, B.R.; Rekow, E.D.; Thompson, V.P. Effect of sandblasting on the long-term performance of dental ceramics. J. Biomed. Mater. Res. Part B Appl. Biomater. 2004, 71, 381-386. [CrossRef] [PubMed]

19. Martins, A.R.M.; Gotti, V.B.; Shimano, M.M.; Borges, G.A.; Goncalves, L. de S. Improving adhesion between luting cement and zirconia-based ceramic with an alternative surface treatment. Braz. Oral Res. 2015, 29, 1-7. [CrossRef] [PubMed]

20. Aboushelib, M.N. Fusion sputtering for bonding to zirconia-based materials. J. Adhes. Dent. 2012, 14, $232-238$.

21. El Gamal, A.; Medioni, E.; Rocca, J.P.; Fornaini, C.; Muhammad, O.H.; Brulat-Bouchard, N. Shear bond, wettability and AFM evaluations on $\mathrm{CO}_{2}$ laser-irradiated CAD/CAM ceramic surfaces. Lasers Med. Sci. 2017, 32, 779-785. [CrossRef]

22. Paranhos, M.P.; Burnett, L.H., Jr.; Magne, M.P. Effect of Nd:YAG laser and $\mathrm{CO}_{2}$ laser treatment on the resin bond strength to zirconia ceramic. Quintessence Int. 2011, 42, 79-89.

23. Cavalcanti, A.N.; Foxton, R.M.; Watson, T.F.; Oliveira, M.T.; Giannini, M.; Marchi, G.M. Bond Strength of Resin Cements to a Zirconia Ceramic with Different Surface Treatments. Oper. Dent. 2009, 34, 280-287. [CrossRef] [PubMed]

24. Stübinger, S.; Homann, F.; Etter, C.; Miskiewicz, M.; Wieland, M.; Sader, R. Effect of Er:YAG, $\mathrm{CO}_{2}$ and diode laser irradiation on surface properties of zirconia endosseous dental implants. Lasers Surg. Med. 2008, 40, 223-228. [CrossRef] 
25. Delgado-Ruíz, R.A.; Calvo-Guirado, J.L.; Moreno, P.; Guardia, J.; Gomez-Moreno, G.; Mate-Sánchez, J.E.; Ramirez-Fernández, P.; Chiva, F. Femtosecond laser microstructuring of zirconia dental implants. J. Biomed. Mater. Res. Part B Appl. Biomater. 2011, 96 B, 91-100. [CrossRef]

26. Akpinar, Y.Z.; Yavuz, T.; Aslan, M.A.; Kepceoglu, A.; Kilic, H.S. Effect of different surface shapes formed by femtosecond laser on zirconia-resin cement shear bond strength. J. Adhes. Sci. Technol. 2015, 29, 149-157. [CrossRef]

27. Tzanakakis, E.; Kontonasaki, E.; Voyiatzis, G.; Andrikopoulos, K.; Tzoutzas, I. Surface characterization of monolithic zirconia submitted to different surface treatments applying optical interferometry and raman spectrometry. Dent. Mater. J. 2020, 39, 111-117. [CrossRef]

28. Prieto, M.V.; Gomes, A.L.C.; Martín, J.M.; Lorenzo, A.A.; Mato, V.S.; Martínez, A.A. The effect of femtosecond laser treatment on the effectiveness of resin-zirconia adhesive: An in vitro study. J. Lasers Med. Sci. 2016, 7, 214-219. [CrossRef] [PubMed]

29. Bärsch, N.; Werelius, K.; Barcikowski, S.; Liebana, F.; Stute, U.; Ostendorf, A. Femtosecond laser microstructuring of hotisostatically pressed zirconia ceramic. J. Laser Appl. 2007, 19, 107-115. [CrossRef]

30. Yucel, M.T.; Kilic, I.; Kilic, H.S.; Gundogdu, Y.; Okutan, Y. Effect of femtosecond laser beam angle and formed shape on surface roughness and shear bond strength between zirconia and resin cement. J. Adhes. Sci. Technol. 2018, 32, 1265-1277. [CrossRef]

31. Vicente, M.; Gomes, A.L.; Montero, J.; Rosel, E.; Seoane, V.; Albaladejo, A. Influence of cyclic loading on the adhesive effectiveness of resin-zirconia interface after femtosecond laser irradiation and conventional surface treatments. Lasers Surg. Med. 2016, 48, 36-44. [CrossRef] [PubMed]

32. Tostes, B.O.; Guimarães, R.B.; Noronha-Filho, J.D.; dos Santos Botelho, G.; Guimarães, J.G.A.; da Silva, E.M. Characterization of conventional and high-translucency Y-TZP dental ceramics submitted to air abrasion. Braz. Dent. J. 2017, 28, 97-104. [CrossRef] [PubMed]

33. Gomes, A.L.; Castillo-Oyagüe, R.; Lynch, C.D.; Montero, J.; Albaladejo, A. Influence of sandblasting granulometry and resin cement composition on microtensile bond strength to zirconia ceramic for dental prosthetic frameworks. J. Dent. 2013, $41,31-41$. [CrossRef]

34. Nagaoka, N.; Yoshihara, K.; Tamada, Y.; Yoshida, Y.; Meerbeek, B. Van Ultrastructure and bonding properties of tribochemical silica-coated zirconia. Dent. Mater. J. 2019, 38, 107-113. [CrossRef]

35. Tzanakakis, E.; Tzoutzas, I.; Kontonasaki, E. Zirconia: Contemporary views of a much talked material: Structure, applications and clinical considerations. Hellenic Stomatolog. Rev. 2013, 57, 101-137.

36. Yagawa, S.; Komine, F.; Fushiki, R.; Kubochi, K.; Kimura, F.; Matsumura, H. Effect of priming agents on shear bond strengths of resin-based luting agents to a translucent zirconia material. J. Prosthodont. Res. 2018, 62, 204-209. [CrossRef] [PubMed] 